\title{
Application of SIMS-SSAMS to Characterization of Surrogate Pre- and Post- Detonation Urban Debris Standard Reference Materials.
}

\author{
Evan E. Groopman ${ }^{1}$, Kenneth S. Grabowski ${ }^{1}$ and Albert J. Fahey ${ }^{2}$ \\ 1. Materials Science and Technology Division, U.S. Naval Research Laboratory, Washington, DC, USA \\ 2. Microscopy \& Surface Analysis, Corning Incorporated, Corning, NY, USA
}

NIST and several partner institutions have undertaken the development of a Surrogate Post-(nuclear)Detonation Urban Debris (SPUD) Standard Reference Material (SRM), see [1]. As no post-detonation urban SRM currently exists, a U-enriched matrix analogous to urban "rubble” is being developed as a predetonation standard, which may subsequently be neutron-irradiated under various known conditions to generate predictable fission products. The pre-detonation standard has been characterized by X-Ray fluorescence, neutron activation, and isotope dilution inductively coupled plasma mass spectrometry. We report here on the application of a globally unique secondary ion mass spectrometer - single-stage accelerator mass spectrometer (SIMS-SSAMS) at the U.S. Naval Research Laboratory (NRL) to the measurement of actinides (fissionable material) and rare earth elements (REE; fission products) in order to support micro-scale characterization of the pre- and post-detonation SPUD SRM.

The NRL SIMS-SSAMS consists of a modified Cameca ims 4f SIMS, which can inject positive or negative ions into a $300 \mathrm{kV}$ National Electrostatics Corp. SSAMS [2]. The combination instrument mitigates drawbacks of each individual technique, providing $\mu \mathrm{m}$-scale lateral resolution from the SIMS with atomic ion specificity (i.e. no molecular isobars due to molecule destruction) from the SSAMS. Since most elements are electropositive, injecting positive secondary ions from the SIMS into the SSAMS greatly enhances measurement sensitivity (impossible for tandem AMS), enabling trace element AMS measurements in particulates. Molecules from the SIMS are dissociated in a gas stripper cell filled with Ar. See [2] for details.

The first reported measurements from the SIMS-SSAMS [2] detailed U isotope ratios and hydride destruction from NIST $\mathrm{U}_{3} \mathrm{O}_{8}$ Certified Reference Material (CRM) particles U850, U500, and U030A (85\%, 50\%, and $3 \%{ }^{235} \mathrm{U}$, respectively). The instrumental bias reported in that paper was later determined to be caused by an improperly installed electrostatic lens on the SSAMS, now corrected. Subsequent measurements of $\mathrm{U}$ isotope ratios in $\mathrm{U}_{3} \mathrm{O}_{8} \mathrm{CRM}$ particles, obtained under similar measurement conditions, all match the certificate values within $1 \sigma$ (Figure 1). The U030A particles measured were all small, with ${ }^{234} \mathrm{U}$ and ${ }^{236} \mathrm{U}$ count rates of $\sim 1$ count/s and $>0.1$ count/s, respectively, compared to $<10,000$ counts/s ${ }^{238} \mathrm{U}$, hence the large $(1 \sigma)$ errors bars. No ${ }^{239} \mathrm{Pu}$ was observed in any sample, indicating complete molecular destruction.

The heavy REE (HREE) fission products from ${ }^{239} \mathrm{Pu}$ and ${ }^{233,235} \mathrm{U}$ differ by orders of magnitude, potentially providing significant leverage for device attribution, but they comprise only $10^{-9}$ to $10^{-6}$ of the fission yield fraction. Conventional SIMS is additionally unable to directly resolve the HREE from light REE (LREE) oxide isobars, requiring careful measurement of element/oxide ratios of all elements and the assumption of terrestrial isotope ratios for deconvolution, e.g. [3]. The latter assumption is incompatible with fission product analysis. Additionally, the setup is complex and time-consuming. With the SIMS-SSAMS, LREE and HREE may be measured directly by eliminating molecular isobars [4]. Figure 2 shows the REE abundance pattern of a monazite $\left((\mathrm{Ce}, \mathrm{La}, \mathrm{Nd}) \mathrm{PO}_{4}\right)$ mineral generously provided by the National Museum 
of Natural History (NMNH, \#147350). Measurements were made with a 1-10 nA O- primary beam focused onto a 20-25 um spot. The left panel shows the CI chondrite normalized abundances [5]; the right panel shows the absolute abundances relative to energy-dispersive X-Ray quantification. Together, these data illustrate our ability to accurately measure the actinide isotopic composition of small, spatially heterogeneous nuclear materials, and to easily measure a wide range of HREE abundances in the presence of large LREE oxide interferences. We plan to apply these capabilities to the characterization of pre- and post-detonation SPUD SRMs for nuclear forensics [6].

\section{References:}

[1] JL Mann et al, Methods and Applications of Radioanalytical Chemistry X (2015), Abs. \#331

[2] A Fahey et al, Analytical Chemistry 88 (2016), p. 7145

[3] AJ Fahey, International Journal of Mass Spectrometry 176 (1998), p. 63

[4] EE Groopman et al, Lunar and Planetary Science XLVIII (2017), Abs. \#1551

[5] E Anders and N Grevesse, Geochimica et Cosmochimica Acta 53 (1989), p. 197

[6] The authors acknowledge the Office of Naval Research for funding and support, and Jackie Mann (NIST) and Richard Essex (NIST/DHS) for helpful discussions.

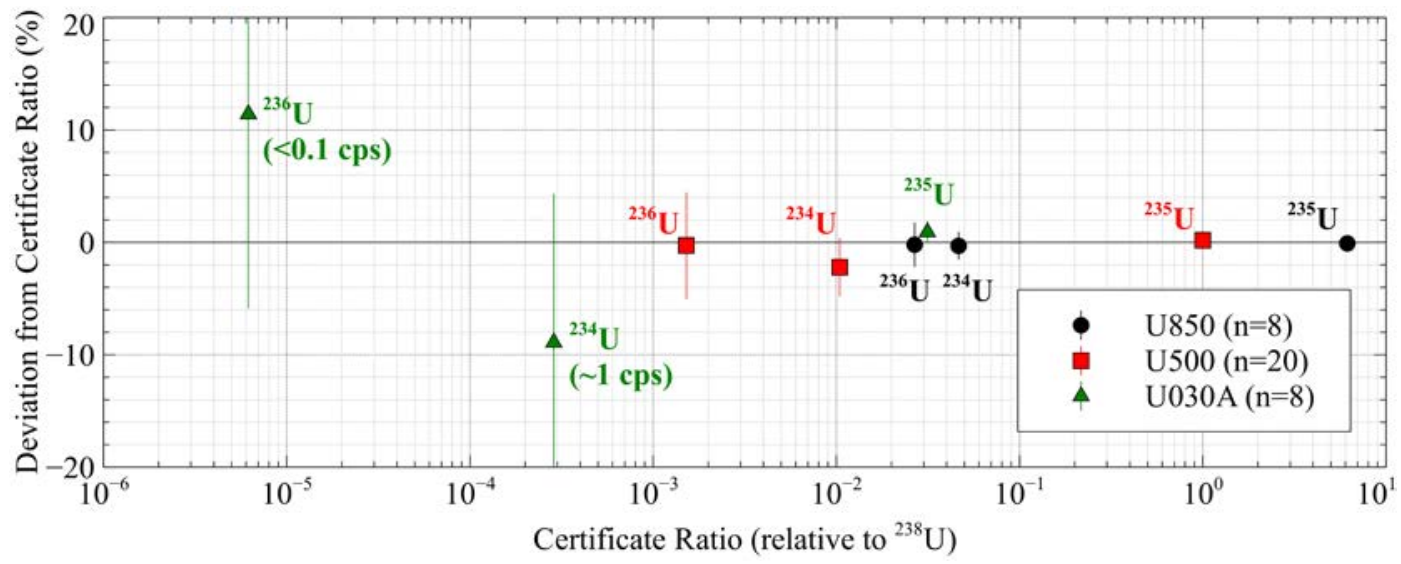

Figure 2: U isotope ratio deviation from certificate value for U850, U500, and U030A particles.
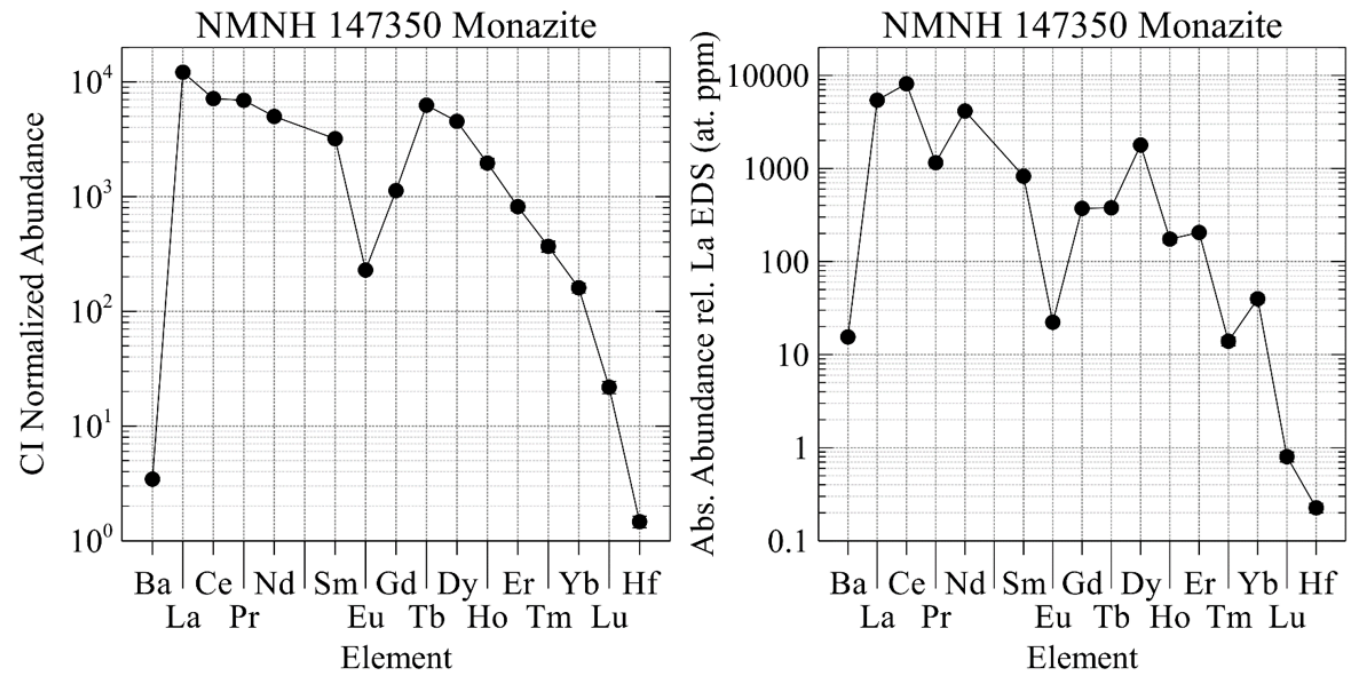

Figure 1: REE abundances in monazite NMNH 147350. (Left) CI chondrite normalized [5], (Right) absolute abundances. Error bars smaller than symbols. 